\title{
Investigation of Streptococcus pyogenes Carriage in Population Vulnerable to Scarlet Fever during 2015-2017 in Shanghai, China
}

\author{
Mingliang Chen ${ }^{1,2}$, Chi Zhang1, Dechuan Kong1, Hao Pan1', Xi Zhang1, Min Chen ${ }^{1 *}$ \\ ${ }^{1}$ Shanghai Municipal Center for Disease Control and Prevention, Shanghai, China \\ ${ }^{2}$ Shanghai Institutes of Preventive Medicine, Shanghai, China \\ Email: *chenmin@scdc.sh.cn
}

How to cite this paper: Chen, M.L., Zhang, C., Kong, D.C., Pan, H., Zhang, X. and Chen, M. (2018) Investigation of Streptococcus pyogenes Carriage in Population Vulnerable to Scarlet Fever during 2015-2017 in Shanghai, China. Journal of Biosciences and Medicines, 6, 89-97.

https://doi.org/10.4236/jbm.2018.611009

Received: October 26, 2018

Accepted: November 16, 2018

Published: November 19, 2018

\begin{abstract}
This study aimed to investigate the carriage of Streptococcus pyogenes in population vulnerable to scarlet fever and to compare their genotypic characterization between different age groups. Pharyngeal swabs were collected from 120 - 150 students in each of the three districts in Shanghai in May and December during 2015 to 2017, while emm typing and detection of 12 superantigen genes were performed to characterize the isolates. During 2015-2017, the average carriage rate in students was $5.7 \%(135 / 2,371)$, without significant difference between different years or districts. The carriage rate was significantly different between children from the three age groups, with $2.4 \%$ in 3 4 years, $5.4 \%$ in 5 - 9 years, and $9.1 \%$ in $10-14$ years. Eight emm types were found, including emm 1, emm 4, emm 12, emm 22, emm 75, emm 89, emm 70 and emm 241, among which emm 12 accounted for 60\%, and emm 1 $27.5 \%$. The predominance of emm 12 was found in each year, but the proportion of emm 12 was lower in $10-14$ years $(43.3 \%)$ than in $3-4$ years $(86.7 \%)$ and in $5-9$ years (73.3\%) $(P=0.002$ and 0.003$)$. Superantigen genes of speB, speC, speG, ssa and smeZ were found in almost all the isolates. The average carriage of $S$. pyogenes in population vulnerable to scarlet fever was $5.7 \%$ in Shanghai, highest in $10-14$ years $(9.1 \%)$, while emm 12 was the predominant type.
\end{abstract}

\section{Keywords}

Scarlet Fever, Carriage Rate, Child, Emm type, Superantigen

\section{Introduction}

Scarlet fever, one of the class B notifiable diseases in China [1], is caused by 
Streptococcus pyogenes. Since 2011, epidemics of scarlet fever have been prevalent in China, with annual reported cases ranging from 34,207 to 68,249 [2]. The epidemics peaked in April to June and November to the next January, and mainly affected children (aged $<15$ years) in kindergarten and school [3]. That is why we supposed school-age children as "population vulnerable to scarlet fever". However, little is known about the $S$. pyogenes carriage in this population. To bridge the gap, this study investigated and analyzed the carriage of $S$. pyogenes in population vulnerable to scarlet fever in Shanghai during 2015 and 2017, which can provide data for preventing and controlling the scarlet fever outbreaks.

\section{Materials and Methods}

\subsection{Carriage Surveys of $S$, pyogenes in Schools}

In May and December, the peak months of scarlet fever epidemics, the carriage surveys were conducted in schools of three districts, including one urban district (Xuhui), one suburban district (Minhang), and one rural district (Fengxian) during 2015 and 2017. The carriage surveys were performed in two schools without scarlet fever clusters within recent one month, and classes without cases were chosen to cover three age groups ( 3 - 4 years, 5 - 9 years, and 10 - 14 years) each with 40 - 50 students. After the participates' parent(s)/guardian(s) gave informed consent, posterior pharyngeal swab samples were obtained by the trained personnel and were sent to Shanghai CDC laboratory in four hours for further test. The study was approved by Shanghai CDC Ethical Review Committee (No.: 2016-4).

\subsection{Strain Collection and Identification}

After culture for 18 - $24 \mathrm{~h}$ on Columbia sheep blood agar at $36^{\circ} \mathrm{C}$ with $5 \%$ carbon dioxide, $\beta$-hemolytic Gram-positive cocci were further tested by latex-agglutination with the Diagnostic Streptococcal Grouping Kit (Oxoid, Hampshire, United Kingdom) and were identified by Vitek 2 system (bioMe'rieux, Marcy l'Etoile, France).

\subsection{Molecular Typing for $S$, pyogenes}

According to the protocol recommended by CDC [4], chromosomal DNA was extracted, emm gene was amplified and sequenced. Nucleotide sequence was blast in the website (https://www2a.cdc.gov/ncidod/biotech/strepblast.asp), and the emm type and subtype were determined. Twelve superantigen genes were screened by PCR as previously reported [5], including spe $A$, speB, speC, speG, speH, speI, speJ, speK, speL, speM, ssa, and smeZ.

\subsection{Statistical Analysis}

Statistical analysis was performed using OpenEpi (Version 3.01) [6]. Statistical significance was assessed at $P<0.05$. Figures were produced using Microsoft 
Excel 2010

\section{Results}

\subsection{Carriage Surveys during 2015 and 2017}

A total of 2371 pharyngeal swabs were collected during 2015 and 2017, and 135 $(5.7 \%)$ were culture-positive. When analyzed by year, the carriage rates were $6.3 \%$ in $2015,5.7 \%$ in 2016 , and $5.1 \%$ in 2017 , respectively (Table 1 ), but the difference showed no statistical significance $(P=0.3-0.6)$. When analyzed by geographical location, the rates were $4.6 \%$ in urban distinct, $5.6 \%$ in suburban district, and $6.7 \%$ in rural distinct, respectively (Table 1), without statistically significant difference $(P=0.08-0.4)$. When analyzed by age, the rates were $2.4 \%$ in 3 - 4 years group, $5.4 \%$ in 5 - 9 years group, and $9.1 \%$ in $10-14$ years group, respectively (Table 2$)$, with statistically significant difference $(P<0.05)$.

\section{2. emm Typs of $S$, pyogenes}

A total of 120 isolates were available for emm typing. There were eight emm types, including emm 1, emm 4, emm 12, emm 22, emm 75, emm 89, emm 170, and emm 241. Isolates of emm 12 were predominant (60\%), followed by emm 1 (27.5\%), while isolates of other types accounted for $12.5 \%$, including emm 4 ( $\mathrm{n}=$ 4), emm $89(\mathrm{n}=4)$, emm $22(\mathrm{n}=3)$, emm $75(\mathrm{n}=2)$, emm $170(\mathrm{n}=1)$, and emm $241(\mathrm{n}=1)$.

Table 1. S. pyogenes carriage in population vulnerable to scarlet fever in three districts of Shanghai, China during 2015 and 2017.

\begin{tabular}{ccccc}
\hline District & 2015 & 2016 & 2017 & Average rate \\
\hline Urban distinct (Xuhui) & $5 \%$ & $4.6 \%$ & $4.2 \%$ & $4.6 \%$ \\
& $(12 / 240)$ & $(11 / 241)$ & $(10 / 240)$ & $(33 / 721)$ \\
Suburban district (Minhang) & $10.2 \%$ & $0.8 \%$ & $5.7 \%$ & $5.6 \%$ \\
Rural distinct (Fengxian) & $(26 / 256)$ & $(2 / 248)$ & $(14 / 246)$ & $(42 / 750)$ \\
& $4 \%$ & $10.7 \%$ & $5.3 \%$ & $6.7 \%$ \\
In total & $(12 / 300)$ & $(32 / 300)$ & $(16 / 300)$ & $(60 / 900)$ \\
& $6.3 \%$ & $5.7 \%$ & $5.1 \%$ & $5.7 \%$ \\
& $(50 / 796)$ & $(45 / 789)$ & $(40 / 786)$ & $(135 / 2,371)$ \\
\hline
\end{tabular}

Table 2. S. pyogenes carriage in population vulnerable to scarlet fever in three age groups in Shanghai, China during 2015 and 2017.

\begin{tabular}{cccccc}
\hline Age group (year) & $\mathbf{2 0 1 5}$ & $\mathbf{2 0 1 6}$ & $\mathbf{2 0 1 7}$ & $\begin{array}{c}\text { Average } \\
\text { rate }\end{array}$ & $\begin{array}{c}\text { Proportion of } \\
\text { emm 12 }\end{array}$ \\
\hline $3-4$ & $\begin{array}{c}3.2 \% \\
(7 / 221)\end{array}$ & $\begin{array}{c}2.6 \% \\
(7 / 266)\end{array}$ & $\begin{array}{c}1.3 \% \\
(3 / 234)\end{array}$ & $\begin{array}{c}2.4 \% \\
(17 / 721)\end{array}$ & $\begin{array}{c}86.7 \% \\
(13 / 15)\end{array}$ \\
& $5.1 \%$ & $4.9 \%$ & $6.2 \%$ & $5.4 \%$ & $73.3 \%$ \\
$5-9$ & $(16 / 315)$ & $(13 / 264)$ & $(18 / 292)$ & $(47 / 871)$ & $(33 / 45)$ \\
& $10.4 \%$ & $9.7 \%$ & $7.3 \%$ & $9.1 \%$ & $43.3 \%$ \\
$10-14$ & $(27 / 260)$ & $(25 / 259)$ & $(19 / 260)$ & $(71 / 779)$ & $(26 / 60)$
\end{tabular}


In each year during 2015 and 2017, emm 12 was the predominant type, and the proportion ranged from $44.4 \%$ to $80 \%$ (Figure 1), with statistically significant difference found between the proportion in 2014 (44.4\%) and that in either of other two years $(P=0.002$ and 0.03$)$. The proportion of emm 12 varied in different age groups, with difference found between the proportion in $10-14$ years group (43.3\%) and that in other two age groups ( $P=0.002$ and 0.003$)$.

There were $21 \mathrm{emm}$ subtypes, including $4 \mathrm{emm} 1$ subtypes and $8 \mathrm{emm} 12$ subtypes. The subtypes emm 12.0 (40\%, 48/120) and emm $1.0(20 \%, 24 / 120)$ were prevalent, while other subtypes accounted for $40 \%$ (48/120). The profile of emm subtypes was not the same in either year during 2015 and 2017, with the number ranging from eight to fourteen (Table 3).

\subsection{Superantigen Profile of $S$, pyogenes}

Among the 120 isolates, all possessed speB, ssa, and smeZ, the majority harbored speC (99.2\%) and speG (98.3\%), almost half possessed speH (54.2\%) and speI (59.2\%), and a few had speA (29.2\%), speJ (12.5\%), speL (2.5\%), and speM (2.5\%), while none had speK (Table 4). In emm 12 isolates, the superantigen profile of speB-speC-speG-speH-speI-ssa-smeZ $Z$ was predominant $(84.7 \%, 61 / 72)$, while in emm 1 isolates, speA-speB-speC-speG-speJ-ssa-smeZ $(39.4 \%, 13 / 33)$ and speA-speB-speC-speG-ssa-smeZ $(33.3 \%, 11 / 33)$ were much common.

\section{Discussion}

China has been undergoing scarlet fever epidemics these years [7]. Based on continuous surveillance on children in school, this study discovered the carriage rate of $S$. pyogenes in population vulnerable to scarlet fever was $5.7 \%$ in peak seasons of scarlet fever, with emm 12 as the predominant type and without difference in geographical districts. Due to no carriage data from other countries with scarlet

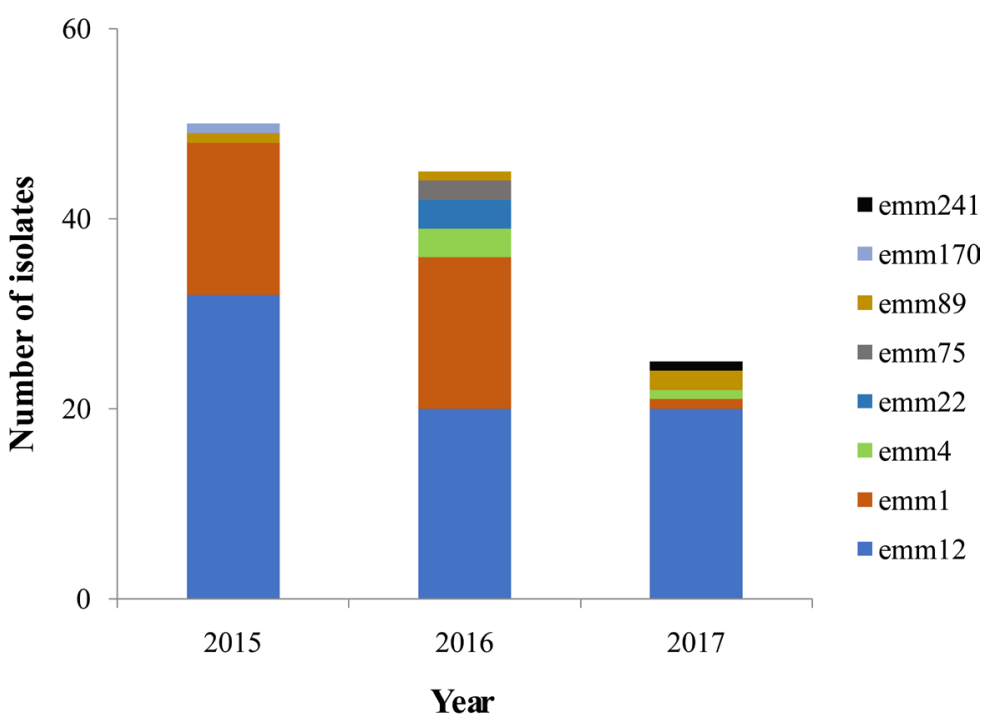

Figure 1. Proportion of emm types of isolates from carriage surveys in Shanghai, China during 2015 and 2017. 
Table 3. Distribution of emm types and subtypes of $S$. pyogenes from population vulnerable to scarlet fever in Shanghai, China, during 2015 and 2017.

\begin{tabular}{|c|c|c|c|c|}
\hline $\begin{array}{l}\text { emm type and } \\
\text { subtype }\end{array}$ & $\begin{array}{c}2015 \\
(\mathrm{n}=50)\end{array}$ & $\begin{array}{c}2016 \\
(n=45)\end{array}$ & $\begin{array}{c}2017 \\
(\mathrm{n}=25)\end{array}$ & $\begin{array}{c}\text { In total } \\
(n=120)\end{array}$ \\
\hline \multicolumn{5}{|l|}{$e m m 1$} \\
\hline 1.0 & $10(20 \%)$ & $14(31.1 \%)$ & 0 & $24(20 \%)$ \\
\hline 1.16 & 0 & $1(2.2 \%)$ & 0 & 0 \\
\hline 1.25 & $4(8 \%)$ & 0 & 0 & $4(3.3 \%)$ \\
\hline 1.65 & $2(4 \%)$ & $1(2.2 \%)$ & $1(4 \%)$ & $4(3.3 \%)$ \\
\hline \multicolumn{5}{|l|}{ emm 12} \\
\hline 12.0 & $21(42 \%)$ & $15(33.3 \%)$ & $12(48 \%)$ & $48(40 \%)$ \\
\hline 12.18 & 0 & $1(2.2 \%)$ & 0 & $1(0.8 \%)$ \\
\hline 12.19 & $1(2 \%)$ & $1(2.2 \%)$ & $1(4 \%)$ & $3(2.5 \%)$ \\
\hline 12.37 & $1(2 \%)$ & $2(4.4 \%)$ & 0 & $3(2.5 \%)$ \\
\hline 12.71 & $1(2 \%)$ & 0 & 0 & $1(0.8 \%)$ \\
\hline 12.76 & $8(16 \%)$ & $1(2.2 \%)$ & 0 & $9(7.5 \%)$ \\
\hline 12.8 & 0 & 0 & $6(24 \%)$ & $6(5 \%)$ \\
\hline 12.95 & 0 & 0 & $1(4 \%)$ & $1(0.8 \%)$ \\
\hline \multicolumn{5}{|l|}{ emm 4} \\
\hline 4.0 & 0 & 0 & $1(4 \%)$ & $1(0.8 \%)$ \\
\hline 4.14 & 0 & $3(6.7 \%)$ & 0 & $3(2.5 \%)$ \\
\hline \multicolumn{5}{|l|}{ emm 22} \\
\hline 22.0 & 0 & $1(2.2 \%)$ & 0 & $1(0.8 \%)$ \\
\hline 22.1 & 0 & $2(4.4 \%)$ & 0 & $2(1.7 \%)$ \\
\hline \multicolumn{5}{|l|}{ emm 75} \\
\hline 75.0 & 0 & $1(2.2 \%)$ & 0 & $1(0.8 \%)$ \\
\hline 75.3 & 0 & $1(2.2 \%)$ & 0 & $1(0.8 \%)$ \\
\hline \multicolumn{5}{|l|}{ emm 89} \\
\hline 89.0 & $1(2 \%)$ & $1(2.2 \%)$ & $2(8 \%)$ & $4(3.3 \%)$ \\
\hline \multicolumn{5}{|l|}{ emm 170} \\
\hline 170.0 & $1(2 \%)$ & 0 & 0 & $1(0.8 \%)$ \\
\hline \multicolumn{5}{|l|}{ emm 241} \\
\hline 241.1 & 0 & 0 & $1(4 \%)$ & $1(0.8 \%)$ \\
\hline
\end{tabular}

fever epidemics were available, such as United Kingdom, South Korea, and Poland [3] [8] [9] [10] [11], we could not compare the carriage rates with them. While comparing with other provinces in China, the carriage rate in this study was higher than another study in Shandong Province $(2.4 \%, 6 / 253)$, but the latter without information of age groups [12].

Analyzed by age, the carriage rate was highest in $10-14$ years group, followed by 5 - 9 years group, then 3 - 4 years, which was not parallel with the scarlet fever incidences in these age groups. Studies on scarlet fever cases in Hong Kong, 
Table 4. Distribution of superantigen genes in $S$. pyogenes from population vulnerable to scarlet fever in Shanghai, China, during 2015 and 2017.

\begin{tabular}{|c|c|c|c|c|c|c|c|c|c|c|c|c|c|}
\hline emm subtype & Total Number & speA & $s p e B$ & speC & speG & speH & speI & speJ & speK & speL & $s p e M$ & ssa & smeZ \\
\hline \multirow{4}{*}{$\begin{array}{l}e m m 12 \\
(\mathrm{n}=72)\end{array}$} & 61 & - & + & + & + & + & + & - & - & - & - & + & + \\
\hline & 8 & - & + & + & + & - & - & - & - & - & - & + & + \\
\hline & 2 & + & + & + & + & - & - & - & - & - & - & + & + \\
\hline & 1 & + & + & + & + & + & + & - & - & - & - & + & + \\
\hline \multirow{5}{*}{$\begin{array}{c}\text { emm } 1 \\
(\mathrm{n}=33)\end{array}$} & 13 & + & + & + & + & - & - & + & - & - & - & + & + \\
\hline & 11 & + & + & + & + & - & - & - & - & - & - & + & + \\
\hline & 5 & + & + & + & + & - & + & - & - & - & - & + & + \\
\hline & 1 & + & + & - & + & - & - & + & - & - & - & + & + \\
\hline & 3 & - & + & + & + & - & - & - & - & - & - & + & + \\
\hline emm 170 & 1 & - & + & + & + & + & + & - & - & + & + & + & + \\
\hline emm 22 & 3 & - & + & + & + & - & - & - & - & - & - & + & + \\
\hline$e m m 241$ & 1 & + & + & + & + & - & - & + & - & - & - & + & + \\
\hline $\mathrm{emm} 4$ & 2 & - & + & + & + & - & - & - & - & - & - & + & + \\
\hline$(\mathrm{n}=4)$ & 2 & - & + & + & - & - & - & - & - & - & - & + & + \\
\hline emm 75 & 2 & - & + & + & + & + & + & - & - & + & + & + & + \\
\hline emm 89 & 3 & - & + & + & + & - & - & - & - & - & - & + & + \\
\hline$(\mathrm{n}=4)$ & 1 & + & + & + & + & - & - & - & - & - & - & + & + \\
\hline In total ${ }^{*}$ & 120 & $35(29.2)$ & $120(100)$ & $119(99.2)$ & $118(98.3)$ & $65(54.2)$ & $71(59.2)$ & $15(12.5)$ & $0(0)$ & $3(2.5)$ & $3(2.5)$ & $120(100)$ & $120(100)$ \\
\hline
\end{tabular}

*the number in bracket stands for the percentage.

Beijing, Jiangsu, and Shanghai showed that scarlet fever mainly affected children younger than 10 years old [3] [5] [8] [13]. This disparity could be explained with factors including bacteria, host, and environment. Firstly, the $S$. pyogenes isolates carried by children younger than 10 years old might be different from those by 10 - 14 years old, in which the proportion of emm 12 isolates was found much lower than that in the 3 - 4 years and the 5 - 9 years groups in this study (Table 2). Moreover, emm $12 \mathrm{~S}$. pyogenes was responsible for the scarlet fever epidemics in China since 2011 [2]. Secondly, the immune system of children younger than 10 years old might be not so mature as that of 10 - 14-year-old children, which makes the former much more vulnerable than the latter when facing the same type of $S$. pyogenes strain. Thirdly, we also thought about the social environment of these groups, but this factor seemed not so important, for children younger than 10 years old and 10 - 14 years old are both in school.

Besides emm typing, the superantigen genes were also studied in population vulnerable to scarlet fever. The genes of $s p e B$, speC, speG, ssa, and $s m e Z$ were found in almost all the emm types, and spec and ssa were supposed as the marker of scarlet fever outbreak strains in China [14]. The profile of superantigen genes, speB-speC-speG-speH-speI-ssa-smeZ, was shared by emm 12 isolates 
from carriers and scarlet fever patients [3], while the similar result was found in emm 1 isolates (speA-speB-speC-speG-speJ-ssa-smeZ), which suggested the scarlet fever epidemics were closely associated with the carriage of epidemic strains in population vulnerable to scarlet fever. Moreover, SpeA, encoded by spe $A$, was supposed as an important virulence factor which facilitated the dissemination of emm 1 strains globally since 1980s due to its ability of enhancing the colonization of $S$. pyogenes in epithelial cells [15]. In previous reports, no spe $A$ gene was found harbored by emm 12 isolates, while in this study, we identify three emm 12 isolates possessing speA gene. This suggested the possibility that emm 12 isolates acquired $s p e A$ gene from emm 1 isolates, which need more data to validate.

\section{Conclusion}

This study provided dynamic data of $S$. pyogenes carriage in school-age children in Shanghai and found the difference of carriage between children in various age groups, which are valuable for prevention and control of scarlet fever epidemics. More studies and surveillance on the carriage in population vulnerable to scarlet fever are needed all over the country.

\section{Funding Statement}

This study was supported by grants from National Natural Science Foundation of China (81601801), Shanghai Rising-Star Program (17QA1403100), a Municipal Human Resources Development Program for Outstanding Young Talents in Medical and Health Sciences in Shanghai (2017YQ039), Natural Science Foundation of Shanghai (16ZR1433300), the $4^{\text {th }}$ Tree-year Action Plan for Public Health of Shanghai (GWTD2015S01) from Shanghai Municipal.

Commission of Health and Family Planning, and the $13^{\text {th }}$ Five-Year Project of National Health and Family Planning Commission of the People's Republic of China (2017ZX10303405004 and 2017ZX10103009-003). The funders had no role in study design, data collection and interpretation, or the decision to submit the work for publication.

\section{Data Availability Statement}

The experimental data used to support the findings of this study are available from the corresponding author upon request.

\section{Conflicts of Interest}

The authors report no conflict of interests regarding the publication of this paper.

The authors alone are responsible for the content and writing of the paper.

\section{References}

[1] Zhang, X., Hou, F., Li, X., Zhou, L., Liu, Y. and Zhang, T. (2016) Study of Surveil- 
lance Data for Class B Notifiable Disease in China from 2005 to 2014. International Journal of Infectious Diseases, 48, 7-13. https://doi.org/10.1016/j.ijid.2016.04.010

[2] You, Y., Davies, M.R., Protani, M., McIntyre, L., Walker, M.J. and Zhang, J. (2018) Scarlet Fever Epidemic in China Caused by Streptococcus pyogenes Serotype M12: Epidemiologic and Molecular Analysis. EBioMedicine, 28, 128-135. https://doi.org/10.1016/j.ebiom.2018.01.010

[3] Yang, P., Peng, X., Zhang, D., Wu, S., Liu, Y., Cui, S., Lu, G., Duan, W., Shi, W., Liu, S., Li, J. and Wang, Q. (2013) Characteristics of Group A Streptococcus Strains Circulating during Scarlet Fever Epidemic, Beijing, China, 2011. Emerging Infectious Diseases, 19, 909-915. https://doi.org/10.3201/eid1906.121020

[4] Streptococcus Laboratory of Centers for Disease Control and Prevention (2017) Introduction to emm Typing: M Protein Gene (emm) Typing Streptococcus pyogenes. https://www.cdc.gov/streplab/groupa-strep/emm-background.html

[5] Chen, M., Yao, W., Wang, X., Li, Y., Wang, G., Zhang, X., Pan, H., Hu, J. and Zeng, M. (2012) Outbreak of Scarlet Fever Associated with emm 12 Type Group A Streptococcus in 2011 in Shanghai, China. The Pediatric Infectious Disease Journal, 31, e158-162. https://doi.org/10.1097/INF.0b013e31825874f3

[6] OpenEpi (2013) Open Source Epidemiologic Statistics for Public Health, Version 3.01. https://www.OpenEpi.com

[7] Liu, Y., Chan, T.C., Yap, L.W., Luo, Y., Xu, W., Qin, S., Zhao, N., Yu, Z., Geng, X. and Liu, S.L. (2018) Resurgence of Scarlet Fever in China: A 13-Year Population-Based Surveillance Study. The Lancet Infectious Diseases, 18, 903-912. https://doi.org/10.1016/S1473-3099(18)30231-7

[8] Lau, E.H., Nishiura, H., Cowling, B.J., Ip, D.K. and Wu, J.T. (2012) Scarlet Fever Outbreak, Hong Kong, 2011. Emerging Infectious Diseases, 18, 1700-1702. https://doi.org/10.3201/eid1810.120062

[9] Park, D.W., Kim, S.H., Park, J.W., Kim, M.J., Cho, S.J., Park, H.J., Jung, S.H., Seo, M.H., Lee, Y.S., Kim, B.H., Min, H., Lee, S.Y., Ha, D.R., Kim, E.S., Hong, Y. and Chung, J.K. (2017) Incidence and Characteristics of Scarlet Fever, South Korea, 2008-2015. Emerging Infectious Diseases, 23, 658-661. https://doi.org/10.3201/eid2304.160773

[10] Turner, C.E., Pyzio, M., Song, B., Lamagni, T., Meltzer, M., Chow, J.Y., Efstratiou, A., Curtis, S. and Sriskandan, S. (2016) Scarlet Fever Upsurge in England and Molecular-Genetic Analysis in North-West London, 2014. Emerging Infectious Diseases, 22, 1075-1078. https://doi.org/10.3201/eid2206.151726

[11] Staszewska, E., Kondej, B. and Czarkowski, M.P. (2014) Scarlet Fever in Poland in 2012. Przeglad Epidemiologiczny, 68, 209-212, 329-231.

[12] Liu, Z., Fang, M., Hu, B., Bi, Z., Kou, Z., Ren, Y., Chen, B. and Bi, Z. (2014) Molecular Types of Group A Streptococcus Isolated from Scarlet Fever Patients and Asymptomatic Carriers in Shandong Province, 2013. Zhonghua Liu Xing Bing Xue Za Zhi, 35, 1375-1378.

[13] Zhang, Q., Liu, W., Ma, W., Shi, Y., Wu, Y., Li, Y., Liang, S., Zhu, Y. and Zhou, M. (2017) Spatiotemporal Epidemiology of Scarlet Fever in Jiangsu Province, China, 2005-2015. BMC Infectious Diseases, 17, 596. https://doi.org/10.1186/s12879-017-2681-5

[14] Davies, M.R., Holden, M.T., Coupland, P., Chen, J.H., Venturini, C., Barnett, T.C., Zakour, N.L., Tse, H., Dougan, G., Yuen, K.Y. and Walker, M.J. (2015) Emergence of Scarlet Fever Streptococcus pyogenes emm 12 Clones in Hong Kong Is Associated with Toxin Acquisition and Multidrug Resistance. Nature Genetics, 47, 84-87. 
https://doi.org/10.1038/ng.3147

[15] Aziz, R.K. and Kotb, M. (2008) Rise and Persistence of Global M1T1 Clone of Streptococcus pyogenes. Emerging Infectious Diseases, 14, 1511-1517.

https://doi.org/10.3201/eid1410.071660 